\section{$\triangle 289$}

TIDAL FLOW VOLUME LOOPS, ECP AND MPO IN SMALL CHILDREN. Karin C. Lødrup Carlsen * Ragnhild Halvorsen **, Kai.H. Carlsen**. * Paediatric dpt., Ullevål Hospital, Oslo. ${ }^{* *}$ Voksentoppen Centre, Osio. Aim:To investigate whether the inflammatory indicators eosinophil cationic protein (ECP) and myeloperoxidase (MPO) in serum reflect possible changes in tidal flow volume (TFV) loops in children with and without obstructive lower airways disease.

Subjects/methods: Blood sampling for serum ECP (Pharmacia CAP, FEIA) and MPO (Pharmacia, RIA) analysis and measurements of TFV loops (SensorMedics 2600) before and 15 minutes after inhaled nebulised salbutamol, were performed in 29 currently asymptomatic, awake children with recurrent bronchopulmonary obstruction (BPO) and 12 awake controls (mean age 12,6 and 12,8 months (n.s.) respectively).

Results: Mean ECP was significantly higher in the sick children $(18,2 \mu \mathrm{g} /)$ than the controls $(9,0 \mu \mathrm{g} /)$ and correlated $(r=0.52)(p<0.001)$ with the magnitude of reversibility to salbutamol in TFV ratios (time/volume to reach peak expiratory flow to total expiratory time/volume). Excluding eczematous children increased the correlation tc $r=0,60$. MPO was higher in the sick children than in the controls (n.s) and correlated with TFV ratios $(r=0.48, p<0.01)$.

Conclusion: Indicators of airways' inflammation (ECP, MPO) were higher in children with recurrent BPO's than in the controls, and correlate with airways' reactivity as measured by TFV loops in awake small children.

\section{$\triangle 292$}

DURATION OF ACTION OF FORMOTEROL AND SȦL̈BUTAMOL DRY-PONDER INHALATION IN PREVENTION OF EXERCISE-INDUCED ASTHMA IN CHILDREN

Kim G.Nielsen, Peter Daugbjerg, Marianne Skov, Hans Bisgaard

Dept.of Raediat.,State University Hospital,Rigshospitalet, Copenhagen The duration and degree of the preventative effect of a single dose formoterol $12 \mu \mathrm{g}$ and salbutamol $400 \mu \mathrm{g}$ dry-powder against exercise induced asthma (EIA) was compared in a randomised, double-blind, placebo-controlled study. Sixteen children (10-14 yrs) with EIA and a maximal fall in FEV1 of 388 (median, range:22-798) were included in the study. On each of 3 different days they inhaled either formoterol $12 \mu \mathrm{g}$ or salbutamol $400 \mathrm{\mu g}$ or placebo dry-powder from an ISF device. A standardized submaximal treadmill exercise challenge test was performed 3 and 12 hours after inhalation. The degree of preventative effect of active drug was calculated as $100 \star$ (EIAP - EIAa)/EIAp 8, where EIAP and EIAa are the maximal median fall in FEV1 on active drug and placebo days respectively. Formoterol afforded 728 ( $p-0.0001)$ preventative effect at 3 hours and $698 \quad(p=0.0001)$ at 12 hours post inhalation compared to 498 (p-0.0001) and 198 (ns) preventative effect when salbutamol was used. At the first exercise test there was no difference between formoterol and salbutamol ( $\mathrm{p}-0.0551)$, while the difference became evident at the second exercise test after which formoterol was superior to salbutamol $(\mathrm{p}-0.0011)$ in degree of preventative effect against EIA. No side effects were observed.

Conclusion: Formoterol 12ug dry-powder inhaled from an ISF device affords a clinically relevant preventative effect against EIA for a duration of at least 12 hours.

\section{$\triangle 290$}

SURPACE ADSORPTION AND MINIMAI SURPACE TENSION OF BRONCHIAI SURFACTANT PREPARATIONS WITH AND WITHOUT EXOGENOUS SURFACTANT IN PULMONARY DISEASES

Ileana Martin-Carrera, Wolfgang Bernhard, Rayaondos Konstantinos, Anne Arning, Christa Acevedo, Horst von der Hardt; Department of Pediatric Pneumonology, Medical School Hannover, FRG.

Few publications have described the activity of surfactant harvested from tracheo-bronchial material of patients with pulmonary diseases using a bubble-surfactometer. We first determined the minimal surface tensions of Exosurf $R$, Alveofact $R$ and dipalmitoylphosphatidylcholine / phosphatidylglycerol $[7: 3 ; \mathrm{mol} / \mathrm{mol}]$ (DPPC/PG), which were 30.3 respectively $30.3 \mathrm{mN} / \mathrm{m}, 11.9 \mathrm{mN} / \mathrm{m}$, and $9.5 \mathrm{mN} / \mathrm{m}$, respectively. Then the influence of Alveofact $R$ and DPPC/PG on surfactant preparations of patients with various pulmorary diseases was measured. surfactant prepaxations or patients were purified from sputum or tracheo-bronchial aspirates by means of a sodium bromide gradient ultracentrifugation (1). Cystic fibrosis patients showed minimal surface tensions of $41 \pm 5 \mathrm{mN} / \mathrm{m}$ compared to $21 \pm 4 \mathrm{mN} / \mathrm{m}$ in other pulmonary diseases. These values were reduced by 50 to $60 \%$ using Alveof act $R$ or DPPC/PG in vitro.

(1) A Method for Reproducible Pulsating Bubble Studies on Surfactant Isolated from Tracheal Aspirates of Infants. Raymondos K, Martin-Carrera I, Bernhard W, C. Acevedo, von der Hardt, H (submitted).

\section{$\Delta 291$}

RESPIRATORY MECHANICHS IN INFANTS WITH ACUTE BRONCHIOLITIS Antonio Martínez-Gimeno, María DolöresG Caballero, Angel Lopez-Silvarrey, José R. Villa-Asensi, Milagros Marin, Gloria García-Hernández, Angel Nogales Department of Paediatrics. University Hospital 12 de Octubre. Madrid. Spain. Objective: to determine alterations in pulmonary function tests in infants in acute phase of bronchiolitis. Subjects and methods: Partial forced expiratory flowvolume curves obtained by an inflatable jacket and end-inspiratory single occlusion test were used to determine maximal flow at Functional Residual Capacity $\left(\mathrm{V}_{\operatorname{mexFRC}}\right)$ and total respiratory system compliance and resistance $\left(C_{n}, R_{r y}\right)$ in 54 previously healthy infants in acute phase of bronchiolitis and in 44 healthy control infants aged 1-12 months. PEDS and PUFF systems (Medical Associates, Hatfield, Pa, USA) were used for these measurements. Results: see table.

\begin{tabular}{|l|c|c|c|c|c|c|c|c|}
\hline Group & $\mathrm{n}$ & age & length & $\mathrm{V}_{\text {maufrc }}$ & \%pred $^{\prime}$ & $\mathrm{C}_{n}$ & $\mathrm{C}_{n} / \mathrm{kg}$ & $\mathrm{R}_{n}$ \\
\hline Bronchiol. & 54 & $0.39(0.2)$ & $62.1(5.6)$ & $55(33)$ & $35(20)$ & $5.2(2.9)$ & $0.79(0.4)$ & $55(21)$ \\
\hline Control & 44 & $0.46(0.1)$ & $64.4(3.0)$ & $190(60)$ & $113(63)$ & $7.5(1.7)$ & $1.0(0.25)$ & $55(14)$ \\
\hline Comp. (p) & & NS & NS & $<10^{8}$ & $<10^{8}$ & $<10^{-8}$ & $<10^{-7}$ & 0.17 \\
\hline
\end{tabular}

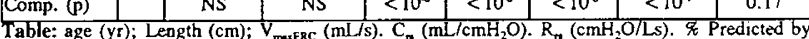

Tepper et al. Am Rev Respir Dis 1986;134:513-9. Mean (Standard deviation). Comparison by $t$ test for independent samples. NS: no significative.

Conclusion: Important decrease in $\mathrm{V}_{\text {ruxfRC }}$ and compliance with no significant increase in resistance characterizes acute phase of bronchiolitis.

\section{$\triangle 293$}

BETWEEN-OBSERVER VARIATION OF A NEW SCORING SYSTEM FOR CF CHEST RADIOGRAPHS

R Oosterkamp, S Robben, F Bröker, C van der Starre, W Hop, JC de Jongste. Depts of Paediatrics, Radiology and Biostatistics, Erasmus University/University Hospital/Sophia Children's Hospital Rotterdam, and Juliana/Lukas Hospital, Apeldoorn, The Netherlands.

Recently a new cystic fibrosis chest radiograph scoring system was introduced ( $\mathrm{J}$ Pediatrics 1993; 91: 488-495), consisting of a weighted assessment of 24 different items for each chest X-ray. The authors stated that this scoring system had a good agreement between observers, and might be useful to assess the severity of lung involvement in young CFpatients. We wanted to confirm between-observer agreement for this complicated scoring system. At the Sophia Children's Hospital, Rotterdam, and the Juliana-Lukas Hospital, Apeldoorn, two experienced independent radiologists were asked to score 70 chest radiographs of 10 cystic fibrosis of 13 years, in random order and blinded. Results: The agreement, as reflected by kappa coefficients of concordance, ranged between -0.04 and 1.00 , but was marginal for most of the 24 items. The bes agreement was reached for uncommon parameters, such as atelectasis. The worst agreement was found for peribronchial thickening, nodular/branching opacities and bronchiectasis.

We conclude that the reproducibility between highly experienced radiologists of this new scoring system was disappointing. This could be overcome by applying more quantitative and strict criteria.

294

EFFECT OF BACTERIAL LYSATE ON LOCAL AND SYSTEMIC IMMUNITY IN BRONCHIECTASIS CHILDREN

Olga I.Orlova, Marina A.Ulanova.

Pulmonology and Immunology Departments, Institute of Pediatrics, Russian AMS, Moscow, Russia.

Prolonged follow-up (to 2 years) of 28 children 3-14 years of age with bronchiectasis due to immunodeficiency and malformations treated with bacterial lysate, Broncho-Munal (BM), has shown clinical improvement in all cases compared to the controls. The study of antibody (Ab) levels to Streptococcus pneumoniae antigens $(\mathrm{Ag})$ in sera, bronchoalveolar lavage fluid (BAL) and saliva in ELISA with the use of capsular polysaccharide, protein $\mathrm{Ag}$ and $C$-polysaccharide as well as immunoglobulins A,M,G demonstrated no significant difference in systemic, local and specific immunity followed by $B M$ treatment in immunocompetent children. At the same time orally administered bacterial antigens caused significant increase in IgA and IgA-Ab levels in sera and secretions in some immunodeficient children. We thus conclude that beneficial clinical effect of BM would be at least partly based on activation of nonspecific defence mechanisms. 